\title{
InteliMed: uma experiência de desenvolvimento de sistema móvel de suporte ao diagnóstico médico
}

\author{
Júlio Venâncio de Menezes Júnior ${ }^{1}$ \\ Raphael José D'Castro ${ }^{2}$ \\ Francisco Marinho Moreira Rodrigues ${ }^{1}$ \\ Cristine Martins Gomes de Gusmão ${ }^{1}$ \\ Nilza Rejane Sellaro Lyra ${ }^{3}$ \\ Silvia Wanick Sarinho ${ }^{3}$
}

\begin{abstract}
Resumo: Atividades de saúde primária e básica normalmente são realizadas por profissionais que possuem conhecimento generalizado, e o serviço oferecido neste contexto é essencialmente descentralizado. Mesmo com a inserção de tecnologias em saúde, bem como a aceitação do uso de equipamentos móveis, percebe-se que ainda há uma lacuna quanto ao uso desses meios em saúde básica, além de se fazerem necessárias ferramentas de apoio à tomada de decisão baseada nas evidências coletadas. Nesse contexto, este artigo apresenta uma proposta e a experiência de construção de sistema de suporte à decisão móvel de apoio ao diagnóstico médico utilizando árvores de decisão.
\end{abstract}

Palavras-chave: Sistema de Apoio à Decisão. Computação Móvel. Saúde Básica.

\begin{abstract}
Primary and basic healthcare activities commonly are made by professionals that have general knowledge, and the service provided in this context is essentially decentralized. Even though the insertion of technologies in healthcare, as well as the acceptance of the usage of mobile devices, there is still a gap regarding the usage of these ones in primary healthcare, and decision-support tools based on collected evidences also are necessary. In this context, this paper presents a proposal and experience of building of mobile decision-support system using decisiontrees.
\end{abstract}

Keywords: Decision-support System. Mobile Computing. Primary Healthcare.

\section{Introdução}

A utilização de dispositivos móveis desempenha papel importante ao dar apoio a serviços de saúde [1], permitindo melhor agilidade desde o processo de coleta de dados até o uso de aplicações que auxiliem no processo de tomada de decisão em seus diversos níveis de complexidade. Também o uso de equipamentos móveis permite a interiorização de aplicações de telessaúde, provendo o acesso descentralizado à informação através redes sem fio [2].

Em saúde comunitária esse cenário não é diferente. Mais especificamente na Estratégia de Saúde da Família (ESF), há uma estrutura organizacional que pode viabilizar a utilização de dispositivos móveis, principalmente devido ao fato de envolver visitas domiciliares. A ESF foi criada pelo governo federal na década

\footnotetext{
${ }^{1}$ Núcleo de Telessaúde da Universidade Federal de Pernambuco, NUTES-UFPE, Pernambuco, Brasil

\{julio.menezes, francisco.rodrigues, cristine.gusmao@nutes.ufpe.br\}

2 Tribunal de Justiça de Pernambuco, TJPE, Brasil

\{raphaeldcastro@tjpe.jus.br\}

${ }^{3}$ Faculdade de Ciências Médicas, Universidade de Pernambuco, FCM-UPE, Brasil

\{nilza.lyra, silviaws@gmail.com\}

doi: $10.5335 /$ rbca. 2011.004
}

Revista Brasileira de Computação Aplicada (ISSN 2176-6649), Passo Fundo, v. 3, n. 1, p. 30-42, mar. 201130 
de 90, ainda com o nome de Programa de Saúde da Família, e promove a saúde básica para populações de baixa renda [3].

A ESF opera com unidades de saúde básicas instaladas com equipes de profissionais que respondem por uma pequena parcela da população (em torno de 2.500 a 4.500 famílias). Em cada unidade há médicos, enfermeiros e agentes comunitários de saúde. $\mathrm{Na}$ maioria dos casos são realizadas visitas domiciliares periódicas, principalmente pelos agentes comunitários de saúde, que funcionam como um elemento facilitador na coleta de evidências para diagnóstico clínico e encaminham a população para o atendimento nas unidades de saúde da família.

A atenção básica à saude requer conhecimento mais geral, sendo necessários contínuos treinamentos e capacitações desses profissionais com a finalidade de torná-los capazes de tomar decisões corretas baseadas em evidências coletadas e referenciar adequadamente os casos não resolvidos na rede básica a níveis hierárquicos mais complexos.

Uma forma de reduzir essa lacuna é utilizar software de apoio ao diagnóstico como recurso de telessaúde. Sistemas de apoio à decisão, por sua vez, atualmente são responsáveis diretos pela melhoria de desempenho de profissionais de saúde [4], e, consequentemente, da qualidade do serviço de saúde oferecido. No entanto, ainda existe uma necessidade de integração do conhecimento agregado sobre diagnóstico e tratamento em sistemas de apoio à decisão clínica para saúde domiciliar [5].

De todos os dispositivos móveis utilizados em saúde, o PDA - Personal Digital Assistant pode ser considerado como o precursor com relação à aceitação do uso entre os profissionais de saúde [6]. Alguns benefícios da sua utilização são: (i) simplificar o acesso e a atualização das informações, tornando-o mais rápido, (ii) fortalecimento da medicina baseada em evidências, (iii) fortalecimento de sistemas de apoio à decisão, (iv) diminuição de erros e inconsistências, (v) utilização como ferramenta de aprendizado entre estudantes e profissionais, dentre outros. Percebe-se que tais benefícios são facilmente estendidos para outros tipos de equipamentos móveis.

Nesse contexto, este artigo apresenta a experiência de modelagem e construção de um modelo de sistema de apoio à decisão descentralizado por meio do uso de dispositivos móveis - InteliMed. O objetivo principal é desenvolver um suporte remoto sobre diagnósticos médicos iniciais com foco em atenção básica à saúde, que inclui o atendimento domiciliar. Inicialmente, para avaliação dos conceitos envolvidos, está sendo desenvolvida uma solução para apoio ao diagnóstico clínico de asma devido à importância da doença para o Sistema Único de Saúde e ao fato de requerer diversos atributos e evidências, muitas vezes despercebidos pelo profissional não especialista.

Após esta seção introdutória, o restante deste artigo está organizado da seguinte forma: a seção 2 discute soluções móveis relacionadas por meio de análise de plataforma e arquitetura utilizadas; a seção 3 introduz aspectos sobre o diagnóstico de asma e trabalhos realizados; a seção 4 faz uma apresentação das necessidades do uso de inteligência computacional como ferramenta de apoio à tomada de decisão nesta; a seção 5 apresenta a proposta do InteliMed e suas peculiaridades; a seção 6, por fim, descreve considerações finais e trabalhos em andamento.

\section{Trabalhos relacionados}

Tecnologias móveis têm sido cada vez mais utilizadas em ambientes de saúde e, desde então, diversos sistemas foram desenvolvidos e modelos propostos. Alguns estudos analisados com o intuito de avaliar a aplicabilidade, bem como questões relativas à arquitetura e a plataformas utilizadas, serão sucintamente descritos a seguir. O objetivo desta revisão foi entender os principais requisitos e fornecer base inicial para a modelagem da solução do InteliMed.

Foram analisados oito estudos disponibilizados na literatura $[7,8,9,10,11,12,13,14]$, selecionados pelo fato de utilizarem soluções móveis para auxílio ao trabalho em saúde, e como resultado foram construídas as Tabelas 1 e 2, que serão apresentadas na subseção 2.1 deste artigo. A seguir, os estudos selecionados são apresentados.

O HandMed (2004) [7], por meio de PDA, permite a detecção prévia de problemas de saúde através de funcionalidade de cadastro de sintomas. Este sistema é voltado para aplicações rotineiras de acompanhamento e pode ser utilizado tanto por pacientes como por profissionais de saúde. O HandMed utiliza a plataforma J2ME 
(Java 2 Micro Edition), e o modelo do PDA utilizado é o Sharp Zaurus SI-5500, que utiliza sistema operacional baseado em Linux com suporte a Java. O sistema é interligado por meio de rede sem fio, que se conecta ao banco de dados do hospital.

O PDAEmbu (2004) [8] é um projeto que desenvolveu um protótipo que utiliza PDA para coletar dados clínicos básicos para o atendimento ambulatorial das Unidades Básicas de Saúde do município de Embu, São Paulo. O formulário de coleta de dados foi construído após entrevistas com estudantes da Universidade de São Paulo (USP). A plataforma escolhida é a J2ME, e a razão para seu uso se deve ao fato de ser livre. A arquitetura é orientada a objetos, visando a futura extensão e reúso. Como o sistema é um protótipo, não foi construído um banco de dados para conexão, mas apenas as interfaces de comunicação para permitir uma possível integração com outros sistemas.

O Clinic Web (2008) [9] integrou o uso de equipamentos móveis para o apoio ao prontuário eletrônico do paciente. Trata-se de um sistema de informação que permite o acesso aos prontuários utilizando PDAs. O módulo móvel do sistema foi desenvolvido utilizando a plataforma MobileVB, que utiliza a linguagem Visual Basic, e possui uma máquina virtual, viabilizando o uso do sistema em diferentes sistemas operacionais. Todas as informações são processadas de maneira off-line, ou seja, não é necessário haver conexão em rede para seu uso. Os dados podem sincronizados com banco de dados através de cabo USB ou conexão Bluetooth.

Outra aplicação proposta faz levantamentos epidemiológicos em saúde bucal, registrando-os em PDA e transformando-os em informações estatísticas com acesso através de Web Services ou transferência TCP/IP por um microcomputador (2008) [10]. Os registros são realizados em aderência à ficha padronizada recomendada pela Organização Mundial de Saúde. Esta aplicação foi desenvolvida utilizando a linguagem C\# e a estrutura de dados para envio e recebimento utiliza o padrão XML (eXtensible Markup Language).

O projeto PEPHans-Pocket (2008) [11] foi desenvolvido para PDA com o objetivo de fornecer melhoria nos serviços prestados à sociedade, acesso simultâneo por profissionais, entre outros. Seu foco é a coleta e o acompanhamento de informações sobre pacientes com hanseníase. O sistema utiliza a linguagem C\#, sendo voltado para ambiente Windows Mobile. O padrão XML é usado para interface de comunicação com servidor, utilizando redes sem fio. Sempre que a sincronização com o banco de dados do servidor é realizada, todos os dados armazenados no PDA são removidos como medida de segurança, e para acesso ao sistema é necessário o uso de nome de usuário e senha. O sistema permite o gerenciamento de cadastros de pacientes, sincronização e controle de notificações incompletas.

O sistema Borboleta (2008) [12] tem um servidor instalado na unidade de saúde que centraliza os dados provenientes das visitas domiciliares e tem o módulo Móvel, que é executado a partir de PDAs durante o atendimento domiciliar para coleta e armazenamento de dados do paciente. A aplicação móvel é voltada para o uso por médicos e enfermeiros e permite a conexão com servidor e banco de dados, bem como a sincronização de dados, uma vez que os dados armazenados no PDA são periodicamente enviados para o servidor. O Borboleta permite o gerenciamento de dados do paciente, agendamento de visitas, acesso ao histórico do paciente, além de disponibilizar um catálogo de doenças e medicamentos.

Já o sistema de informação UTInfo2.0 (2008) [13] foi desenvolvido com o objetivo de permitir o acesso ao Registro Eletrônico de Paciente (REP) por meio de aplicações desktop ou remotamente, via dispositivos móveis. Sua aplicação é voltada para pacientes em unidades de terapia intensiva com o objetivo de facilitar os atendimentos e casos considerados mais críticos, sendo voltado para médicos e enfermeiros. Todo o sistema é baseado na linguagem Java, no qual a aplicação móvel utiliza a plataforma J2ME, também é composto por uma aplicação desktop, que funciona como servidora, utilizando recursos web para as transações entre ambas as aplicações. O padrão de troca de informação entre dispositivos é realizado através de XML, que é usado de forma segura utilizando o protocolo HTTPS. Os autores não mencionam explicitamente o tipo de dispositivo utilizado, mas reforçam que a escolha da plataforma J2ME se deve ao fato de que o desenvolvimento da aplicação para diferentes dispositivos permite a sua utilização sem se preocupar que o tipo do dispositivo é voltado para consulta de registro.

Outro sistema estudado foi desenvolvido para monitorar pacientes remotamente através da aplicação de dispositivos móveis e Web Services (2008) [14]. Também foi proposta uma arquitetura modular que permitisse a integração de diferentes tecnologias e plataformas. A aplicação utiliza a plataforma J2ME e o aparelho utilizado é o celular Nokia 6220, modelo S60. O software móvel conecta-se a um servidor de aplicação em tempo real, que recebe os dados no formato XML, os quais também ficam armazenados em banco de dados para análises futuras.

Revista Brasileira de Computação Aplicada (ISSN 2176-6649), Passo Fundo, v. 3, n. 1, p. 30-42, mar. 201132 
A seguir será apresentada uma análise tecnológica dos trabalhos estudados. Inicialmente, serão vistas questões de tipo de dispositivo e plataforma utilizados e, em seguida, será realizada uma análise do modelo arquitetural móvel.

\subsection{Análise tecnológica}

Com o estudo dos trabalhos relacionados foi possível realizar análise comparativa observando as plataformas móveis utilizadas por cada um, bem como as respectivas arquiteturas. A Tabela 1 apresenta levantamento das características: plataforma e tipo de dispositivo. Para plataforma entende-se como a tecnologia base para o desenvolvimento e execução de aplicações em dispositivos móveis.

Tabela 1. Plataformas e tipos de dispositivo utilizados nos projetos

\begin{tabular}{lll}
\hline Projeto & Plataforma & Dispositivo \\
\hline HandMed [7] & J2ME & PDA \\
PDAEmbu [8] & J2ME & PDA \\
Clinic Web [9] & Mobile VB & PDA \\
Levantamento Epidemiológico [10] & Windows Mobile & PDA \\
PEPHans-Pocket [11] & Windows Mobile & PDA \\
Borboleta [12] & J2ME & Celular / Smartphone / PDA \\
UTInfo 2.0 [13] & J2ME & Celular / Smartphone / PDA \\
Monitoramento Remoto [14] & J2ME & Celular \\
\hline
\end{tabular}

Observa-se que a maioria dos projetos usou a plataforma J2ME, que utiliza a linguagem de programação Java, e o dispositivo PDA. No entanto, atualmente, os celulares evoluíram em questão de processamento de hardware e memória com redução de custo, sendo chamados de smartphones. Inclusive, com a evolução de tais famílias de equipamentos, surgiram novas plataformas que visam otimizar os seus recursos, provendo uma interface mais amigável com o usuário. Dentre as plataformas de smartphones, atualmente podemos destacar: Android (linguagens: Java ou C++), Windows Phone (linguagem: C\#) e iOS (linguagem: Objective-C).

Para a análise da arquitetura, de acordo com Lee e demais autores [15], a arquitetura de uma aplicação móvel caracteriza-se da seguinte forma:

- Cliente-Servidor: Camadas, Filas;

- Clientes: Clientes magros; Clientes gordos; Hospedagem de página web;

- Servidor: Arquitetura de uma fila (one-tier); Arquitetura de duas filas (two-tier); Arquitetura de três filas (three-tier);

- Tipos de Conexão: Sempre conectado; Parcialmente conectado; Nunca conectado;

- Sincronização: Comunicação contínua (Síncrona ou Assíncrona); Armazena e encaminha.

A Tabela 2 traz uma visão do estudo dos principais pontos de arquitetura de aplicação, baseada no levantamento bibliográfico mencionado anteriormente. Vale ressaltar que nem todos os padrões descritos nos estudos avaliados estavam explícitos nos artigos. Portanto, inferiram-se algumas das características, apresentadas na Tabela 2, baseadas nos outros componentes de cada projeto.

Tabela 2. Arquitetura de Aplicação dos Projetos

\begin{tabular}{llllll}
\hline Projeto & Cliente-servidor & Cliente & Servidor & Conexão & Sincronização \\
\hline HandMed & Camada & Gordo & Duas filas & Sempre & Contínua \\
PDAEmbu & Camada & Magro & Duas filas & Sempre & Contínua \\
Clinic Web & Fila & Gordo & Duas filas & Parcial & $\mathrm{A} / \mathrm{E}^{*}$ \\
Levantamento Epidemiológico & Camada & Gordo & Duas filas & Parcial & $\mathrm{A} / \mathrm{E}^{*}$ \\
PEPHans-Pocket & Fila & Gordo & Duas filas & Parcial & $\mathrm{A} / \mathrm{E}^{*}$ \\
Borboleta & Fila & Gordo & Duas filas & Sempre & Contínua \\
UTInfo 2.0 & Fila & Gordo & Duas filas & Parcial & $\mathrm{A} / \mathrm{E}^{*}$ \\
Monitoramento Remoto & Fila & Gordo & Duas filas & Parcial & $\mathrm{A} / \mathrm{E}^{*}$ \\
\hline
\end{tabular}

* A/E = Armazena e encaminha 
Nos projetos percebe-se a predominância no uso de arquitetura cliente-servidor em fila, cliente gordo, servidor em duas filas, conexão parcial do cliente com o servidor e sincronização do tipo armazenar e encaminhar.

Fazendo uma análise geral sobre os dados obtidos de cada trabalho, percebe-se que o uso de fila é positivo, pois facilita a capacidade de escalonamento das aplicações. Um cliente gordo tem a vantagem de operar independentemente do servidor por determinado período, porém ele depende mais do sistema operacional e do tipo de dispositivo móvel do que um cliente magro.

A arquitetura de servidor não está explícita nos trabalhos relacionados; portanto, o padrão de duas filas foi considerado pelo fato de estar em um meio-termo em relação ao de uma fila e de três filas quando se trata de escalonamento, segurança, gerenciamento e custo. Em outras palavras, o servidor de duas filas contempla uma base de dados e uma aplicação que gerencia esta base, enquanto a interface com o usuário fica no lado cliente do sistema. Já o tipo de conexão parcialmente conectado, predominante entre os trabalhos, possibilita que o dispositivo se conecte periodicamente a um servidor para trocar dados, e também pode operar em vários momentos sem haver a necessidade da conexão ao servidor.

Com respeito à sincronização, prevaleceu a do tipo armazenar e encaminhar, que é possível pelo fato de a conexão ser parcial, o que demanda um banco de dados local para armazenamento que encaminhará os dados para o banco do servidor quando a conexão for estabelecida.

A análise da arquitetura em equipamentos móveis foi de suma importância para a modelagem da solução proposta neste trabalho, pois permitiu uma visão mais ampla dos trabalhos relacionados, de forma a projetar o sistema proposto de maneira aderente à visão de arquiteturas móveis existentes. Desse modo, parte-se da premissa de que o software hoje é visto como um serviço; logo, a sua aplicação em diferentes ambientes, contextos e equipamentos precisa ser levada em consideração, permitindo a adaptabilidade futura.

\section{Diagnóstico de asma}

A asma é uma das doenças crônicas mais comuns em todo o mundo e atinge cerca de $20 \%$ de crianças e adolescentes brasileiros [16]. Possui caráter inflamatório e cursa com episódios recorrentes de sibilância (chiado), reversíveis espontaneamente ou com tratamento. Anualmente ocorrem cerca de 350.000 internações por asma no Brasil pelo Sistema Único de Saúde (2,3\% do total), a um custo de cerca de 76 milhões de reais, o terceiro maior valor gasto com uma única doença [16]. A atenção básica à saúde, que é a principal porta de entrada dos pacientes no Sistema Único de Saúde através da ESF, desempenha uma importante função no diagnóstico e tratamento corretos de doenças. Desse modo, é possível contribuir para a diminuição da ocorrência de casos graves e internações hospitalares com elevado custo. As principais manifestações clínicas da asma são dispneia, aperto no peito e tosse. O diagnóstico da doença é baseado em história médica e confirmado pela limitação variável ao fluxo aéreo, demonstrado pela espirometria, pelos testes alérgicos, além do pico de fluxo expiratório e outros exames. O diagnóstico clínico da asma na atenção básica à saúde é importante para iniciar corretamente o tratamento.

O diagnóstico clínico da asma está fundamentado nos seguintes atributos ou aspectos clínicos: dispneia ou falta de ar; tosse crônica ou persistente; sibilância ou chiado no peito; aperto no peito ou desconforto; tosse durante ou após atividade física; sintomas ao acordar/ao se deitar; sintomas episódicos; sintomas sazonais (variações de temperatura); três ou mais sintomas no último ano; melhora espontânea; melhora com medicação (broncodilatadores e/ou corticosteroides); história familiar positiva de atopia (pais e/ou irmãos alérgicos); sintomas desencadeados por alérgenos: mofo, poeira doméstica ou animais; sintomas desencadeados por irritantes: cigarro, perfumes; outros desencadeantes: resfriados, emoções (choro/riso); teve ou tem outras alergias: rinite e dermatite atópica.

Diversos estudos sobre a asma têm sido publicados no Brasil e no exterior contendo normatizações de condutas, que são os guidelines $[17,18,19,20,21,22,23,24]$. Esses estudos visam à ampla difusão do conhecimento sobre o tema e a uma correta abordagem diagnóstica com vistas ao tratamento adequado. As principais fontes disponíveis são: IV Diretrizes Brasileiras para o Manejo da Asma (Consenso Brasileiro) [17]; questionário do ISAAC ("International Study of Asthma and Allergies in Childhood") [23] e consenso GINA

Revista Brasileira de Computação Aplicada (ISSN 2176-6649), Passo Fundo, v. 3, n. 1, p. 30-42, mar. 201134 
(Global Iniative for Asthma) [24], além de outros. Todos advêm de revisões sistemáticas, considerando-se evidências científicas relevantes (medicina baseada em evidências).

\subsection{Proposta de formulário para triagem diagnóstica sobre asma}

A construção de uma árvore de decisão clínica para o diagnóstico de asma com base nas diretrizes atuais pode ser interpretada numa perspectiva clínica. Cinco aspectos dentre os seguintes fatores preditivos foram utilizados como atributos: se já teve asma, asma no último ano, fôlego curto, atopia (predisposição genética para a alergia), chiado e falta de ar. Esses são indícios de considerável sucesso em estudos epidemiológicos anteriores que vêm confirmar o diagnóstico clínico da asma para, assim, proceder-se ao tratamento. Assim, os atributos da asma e, posteriormente, as orientações terapêuticas, contidos em um dispositivo móvel, como ferramenta para o médico generalista, possibilitarão a adesão à orientação dos consensos atuais, o que resultará em potencial melhora na qualidade do tratamento oferecido aos asmáticos.

Como módulo integrante do projeto InteliMed, a equipe de medicina desenvolveu questionário para triagem diagnóstica de asma que contivesse as normatizações dos guidelines com o objetivo de melhorar o atendimento na área de saúde por facilitar o acesso à informação por parte dos profissionais de saúde. Os questionários são instrumentos de pesquisa que devem englobar o maior número de informações sobre o tema, todas embasadas, primordialmente, na abordagem clínica e não funcional. A elaboração do formulário passou por validação inicial de face, que constou da submissão a um comitê de especialistas em pneumologia e alergologia do Centro de Pesquisas em Alergia e Imunologia Clínica da Universidade Federal de Pernambuco. A validação de face feita pelos pares visa à análise do instrumento de pesquisa nos aspectos semântico, na compreensão para o público leigo, além da abrangência do tema a ser pesquisado. As validações de construto e conteúdo são realizadas à medida que se selecionam os fatores de entrada para a árvore de decisão, mediante a colocação de novos atributos com maior ganho de informações. Durante e também paralelamente à elaboração das questões, foram realizadas várias revisões no formulário.

Os questionários foram aplicados aos pacientes atendidos em serviços ambulatoriais de alergia ("casos") e de pediatria e adolescência ("controles"). Foram considerados critérios de inclusão para os pacientes o diagnóstico médico de asma, mediante quadro clínico compatível, descritos nos prontuários.

\section{Inteligência computacional aplicada ao suporte ao diagnóstico médico}

A Inteligência Computacional é uma área da Ciência da Computação que estuda a elaboração e utilização de técnicas com inspiração na natureza ou no comportamento humano. Aspectos como aprendizado, percepção, evolução e adaptação são aplicados em sistemas computacionais, ampliando, assim, as suas potencialidades. O conjunto de técnicas com capacidade de "aprender" é conhecido como Aprendizagem de Máquina (Machine Learning). Essas técnicas possibilitam a identificação de padrões e relações entre os dados, criando, assim, a capacidade de visualização de tendências através do tempo. Nessa perspectiva, as técnicas de Aprendizagem de Máquina podem ser utilizadas na predição e identificação de relações em dados não triviais.

No acervo de técnicas de Aprendizagem de Máquina consta o conjunto de ferramentas que utilizam algoritmos de aprendizagem baseados em estatística ou redes neurais artificiais. Essas técnicas possibilitam a exploração de grandes conjuntos de dados, extraindo ou possibilitando a descoberta de conhecimento. Esse conhecimento pode ser apresentado por essas ferramentas na forma de agrupamentos, hipóteses, regras, árvores de decisão ou grafos. Neste projeto destacamos a Árvore de Decisão devido a sua aplicabilidade ao domínio do diagnóstico em saúde em razão da sua natureza de apoio à decisão com base na inferência em dados coletados em paralelo com histórico de casos passados.

Árvores de Decisão são modelos estatísticos para classificação e predição de dados. Esses modelos utilizam a estratégia "dividir e conquistar" [25], onde um problema complexo é decomposto em subconjuntos mais simples e, recursivamente, esta técnica é aplicada para cada subproblema [26]. Um dos primeiros algoritmos desenvolvidos foi o ID3. Seu conceito de criação usou a ideia dos sistemas de inferência e dos conceitos de aprendizagem de máquina. Em pouco tempo outros algoritmos surgiram: C4.5, CART (Classification and Regression Trees), CHAID (Chi Square Automatic Interaction Detection) e outros [27]. A Figura 1 ilustra uma árvore de decisão, onde são identificados os seguintes elementos:

- Nós (elipses) - são os atributos consideradas no problema (outlook, humidity e windy);

Revista Brasileira de Computação Aplicada (ISSN 2176-6649), Passo Fundo, v. 3, n. 1, p. 30-42, mar. 201135 
- Arcos (testes) - a partir do valor do atributo define qual caminho seguir na árvore (Ex.: Se outlook=sunny, então seguir para humidity);

- Folhas (retângulos) - indica a classe associada aos Nós;

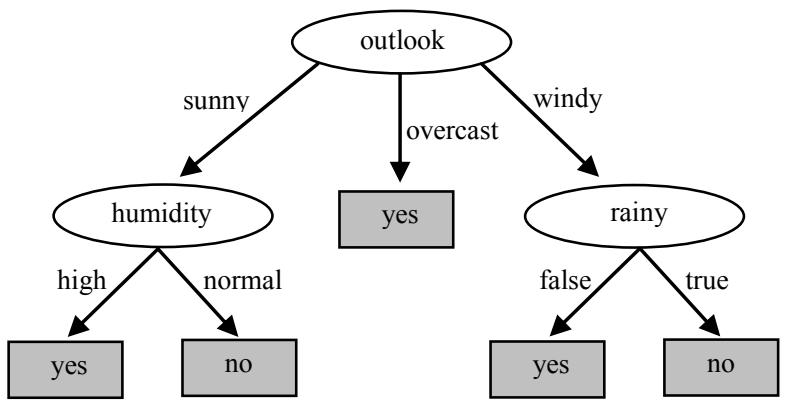

Figura 1. Exemplo de Árvore de Decisão [25]

A seleção de algoritmos que implementam árvores de decisão considera vários aspectos. Entre todos a serem ponderados, alguns se destacam pela sua importância, sendo: (i) o critério de escolha dos atributos a ser utilizado em cada nó, (ii) a forma de calcular o particionamento do conjunto de exemplos a ser utilizado, (iii) a determinação de um nó como folha, (iv) a determinação do critério a ser utilizado na seleção da classe a ser atribuída a cada nó e (v) a aplicação do processo de poda [28].

A grande aplicabilidade das árvores de decisão e sua crescente utilização, tanto na área acadêmica como em aplicações comerciais, dá-se pela sua flexibilidade, robustez, poder de interpretação e velocidade de processamento [29]. Entre as aplicações se destacam:

- medicina, na determinação de diagnóstico e tratamentos, no controle de gastos hospitalares etc.;

- $\quad$ astronomia, em observações atmosféricas, em análise de informações espaciais etc.;

- $\quad$ engenharia, no diagnóstico em automóveis etc.;

- $\quad$ agricultura, na identificação de doenças em produções agrícolas;

- $\quad$ finanças, na detecção de fraudes em seguros, na detecção de uso indevido de cartões de crédito, na aprovação de crédito etc.;

- marketing, na predição de vendas, na classificação de grupos econômico-sociais por comportamento, de acordo com particularidades etc.

O algoritmo escolhido para geração das árvores de decisão no InteliMed foi o C4.5. Este algoritmo se mostra adequado ao projeto, uma vez que possibilita a utilização de atributos categóricos e valores desconhecidos. O fluxo de informações da árvore de decisão proposta para o InteliMed está ilustrado na Figura 2 e pode ser dividido em três etapas: (i) geração da árvore de decisão inicial (treinamento, validação e testes), (ii) geração continuada de novas árvores de decisão (treinamento validação e testes) e (iii) sugestão de diagnóstico (predição).

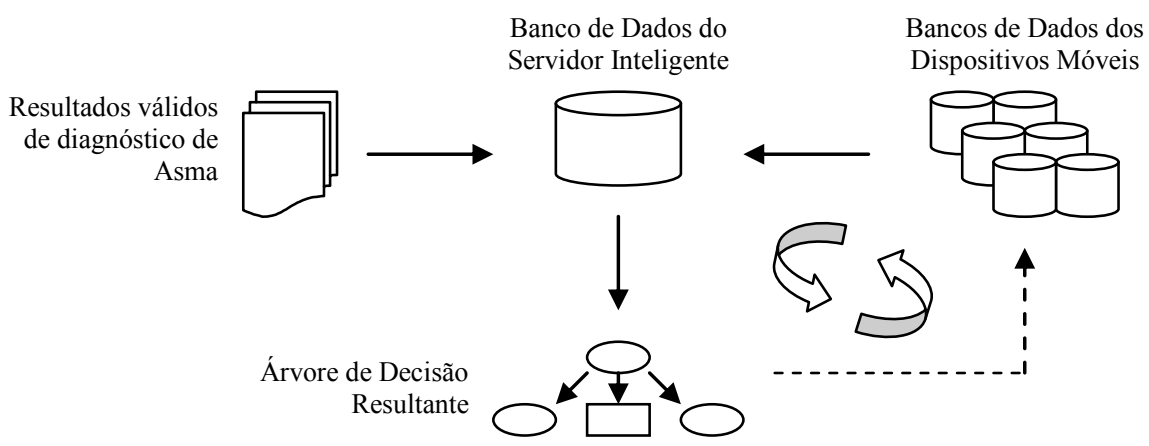

Figura 2. Fluxo de informação da árvore de decisão no InteliMed 
A primeira etapa consiste na coleta dos dados de diagnóstico de asma na rede de saúde de Pernambuco, a partir do questionário introduzido na seção 3.3, visando à obtenção de um histórico representativo do problema. Os dados obtidos nesta etapa serão pré-processados para eliminar diagnósticos com informações insuficientes ou inconsistentes. Após a obtenção de um conjunto significativo de diagnósticos de asma, esses dados serão inseridos na Base de Dados do Servidor Inteligente. Nesse momento será rodado o processo de geração da árvore de decisão inicial, que resultará de uma série de treinamentos, validações e testes a fim de obter uma árvore com alto índice de sucesso nos testes. Uma vez definida, a árvore inicial será distribuída para os dispositivos móveis. Após a conclusão desta etapa, o sistema já está apto a produzir as primeiras sugestões de diagnósticos.

A próxima etapa no fluxo de informação da árvore de decisão visa ao aprimoramento constante com base nas informações obtidas pela utilização da ferramenta. Os dispositivos móveis fornecerão ao Servidor Inteligente informações referentes aos dados dos pacientes, o resultado sugerido pela árvore de decisão e o diagnóstico real apontado pelo profissional de saúde. Essas informações retroalimentarão o sistema de modo a gerar novas árvores de decisão com as novas informações, as quais serão distribuídas aos dispositivos móveis sempre que os testes apontarem resultados superiores à vigente.

A última etapa do ciclo de vida da árvore de decisão consiste na utilização da árvore de decisão vigente para obtenção de uma sugestão de diagnóstico. Esta etapa ocorre exclusivamente no dispositivo móvel, onde os dados do questionário aplicado ao paciente serão utilizados como entrada da árvore de decisão. Como mencionado anteriormente, esses dados e o próprio resultado processado pelo dispositivo móvel retornam para o servidor inteligente para alimentar o processo contínuo de melhoria do sistema.

\section{Proposta do InteliMed}

Com base nos trabalhos estudados, foi elaborada a solução proposta, cuja macrovisão da arquitetura está apresentada na Figura 3. Três módulos compõem o InteliMed: a Aplicação Móvel e os Servidores Intermediário e Inteligente. Para sua construção, o InteliMed conta com equipes distribuídas e multidisciplinares, pois envolve estudantes e profissionais de tecnologia da informação na concepção do sistema, bem como profissionais de saúde na questão de material técnico e clínico de apoio.

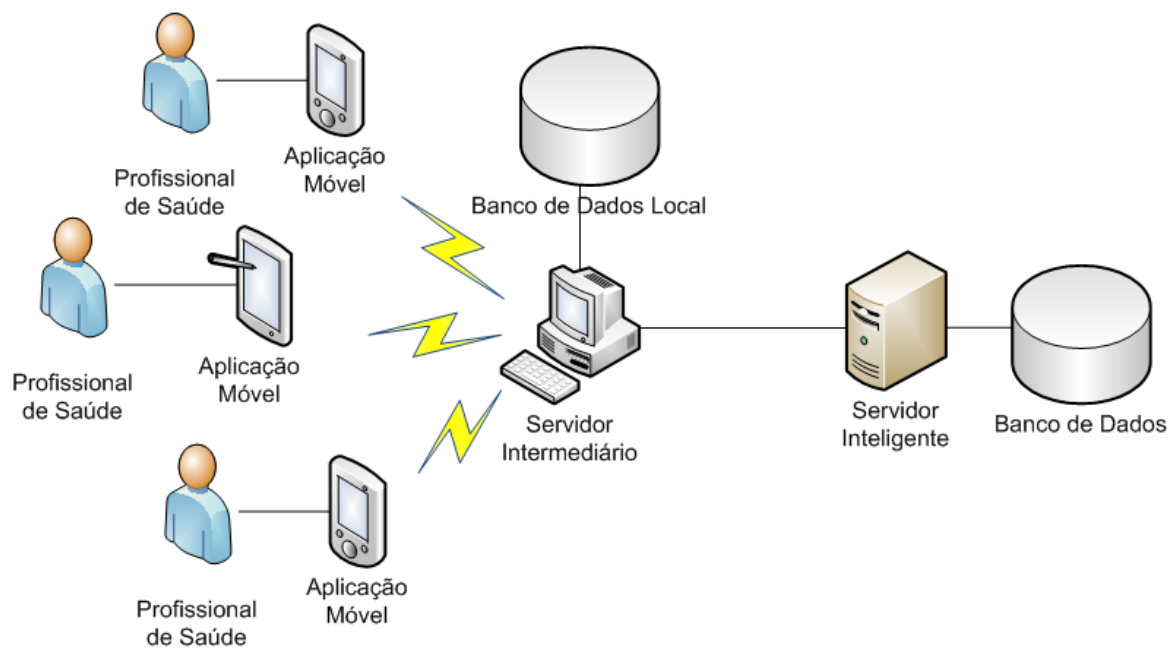

Figura 3. Arquitetura do InteliMed

O módulo de Aplicação Móvel é o responsável por permitir operações de campo, incluindo cadastro de pacientes, diagnósticos e visualização das visitas por funcionário. O Servidor Intermediário é a interface entre a Aplicação Móvel e o Servidor Inteligente, possuindo um banco de dados local e permitindo a sincronização de dados entre ambos. O Servidor Inteligente executa a técnica da construção e modelagem de árvore de decisão sobre uma base de dados consolidada de pacientes em uma determinada especialidade médica.

Com relação à visão global da arquitetura proposta da solução, possui as seguintes características: 
- modelo cliente-servidor: utiliza o modelo em Filas, ou seja, toda a aplicação não está concentrada apenas em um equipamento, e sim distribuída entre o equipamento móvel e servidores;

- cliente é gordo, possibilitando à aplicação móvel menos dependência do servidor;

- servidor Intermediário e o Inteligente adotam a arquitetura de duas filas; logo, cada servidor está separado dos respectivos banco de dados;

- tipo da conexão é parcial, pois a aplicação móvel só precisará estar conectada ao servidor intermediário em dois momentos: no momento da autenticação e ao encerrar o sistema;

- sincronização é do tipo armazena e encaminha; logo, o equipamento móvel possui um banco de dados local que é alimentado constantemente. Ao encerrar o sistema, todos os dados deste banco local são enviados ao servidor intermediário.

Dessa forma, a adequação da arquitetura da solução proposta aos diversos trabalhos relacionados facilitará a disseminação do uso de equipamentos móveis em saúde de uma maneira mais padronizada. Mesmo com diferenças entre a proposta do InteliMed, que é focado no apoio à decisão, e os trabalhos relacionados, vale ressaltar que na realidade está sendo concebido não só um módulo de decisão, mas, sim, um sistema de informação baseado em dispositivos móveis. Desse modo, com essa visão podem-se extrair características de outros sistemas existentes para a construção do sistema proposto.

Devido ao fato de o InteliMed ser uma proposta de sistema de suporte à decisão para saúde básica e também domiciliar, o sistema foi projetado para ser executado de maneira remota, pois o serviço realizado pelos profissionais que fazem parte ESF é essencialmente descentralizado. Vale ressaltar que a conexão entre a Aplicação Móvel e o Servidor Intermediário não é em tempo real e não necessita haver conexão sempre, pois a rede em áreas de população carente normalmente é limitada de recursos, ou até mesmo inexistente.

Baseado na visão geral da ESF, nos modelos arquiteturais dos sistemas estudados, bem como na forma como os dados de atributos da asma podem ser processados, o projeto da solução do InteliMed foi construído. Nas subseções seguintes são descritos cada módulo e suas respectivas funcionalidades.

\subsection{Aplicação móvel}

A aplicação móvel - cliente principal do sistema que inclui todas as operações necessárias para utilização em campo - é implementada utilizando a linguagem de programação Java. Um grande número de dispositivos móveis oferece suporte para esta linguagem, além de ser orientada a objetos, livre e portável. Seguindo a tendência do uso de smartphones, a aplicação móvel do InteliMed está baseada na plataforma Android, que é livre, de código aberto, baseada no núcleo Linux, além de permitir o desenvolvimento de aplicativos utilizando as linguagens Java e $\mathrm{C}++$. As principais funcionalidades da aplicação móvel são:

- autenticar profissional de saúde: a autenticação é realizada pela sincronização dos dados de usuário com o servidor intermediário. Isso permite maior segurança e restrições de acesso para diferentes perfis de usuário;

- gerenciar pacientes: apresenta um formulário que permite o registro de pacientes. Este formulário tende a ser muito simples e possui apenas dados pessoais. Quando o paciente já existe, a aplicação mostra o histórico de visitas e os diagnósticos anteriores realizados com o paciente em questão;

- gerenciar visitas e consultas: permite ver o agendamento de visitas e consultas, assim como registrar consultas futuras - seja na unidade de saúde, seja na casa do paciente;

- reportar suspeita: os agentes de saúde, por serem impedidos de realizar diagnósticos, registram no sistema a suspeita de doença de determinado paciente para que os médicos da unidade de saúde sejam informados;

- determinar diagnóstico: ao responder a um questionário exibido na tela, o médico estará coletando evidências, as quais serão utilizadas pela aplicação móvel para determinar o diagnóstico. A árvore de decisão é percorrida de acordo com as respostas do questionário e, concluído o processamento, o resultado é informado ao usuário. Após exibir o diagnóstico, o aplicativo questiona se o médico concorda, ou não, e possibilita a inserção de comentários adicionais; 
- encerrar sistema: o sistema é sincronizado com o servidor intermediário objetivando enviar todas as informações coletadas durante sua execução e esvaziar a memória interna do dispositivo por questões de segurança.

\subsection{Servidor intermediário}

Este módulo está presente em cada unidade de saúde, possui uma interface de usuário baseada em tecnologia web e é implementado utilizando Java Enterprise Edition - versão de Java destinada para aplicações de servidor. As principais funcionalidades do servidor intermediário são:

- autenticar dispositivo móvel: o servidor recebe os dados de autenticação do usuário e responde com mensagens de autenticação - as quais validam e informam os privilégios do usuário;

- armazenar informações sobre diagnósticos e pacientes: informações sobre os pacientes visitados e seus diagnósticos são armazenados em um banco de dados local quando enviados pelo dispositivo móvel;

- distribuir questionários e árvores de decisão: o servidor envia arquivos contendo o questionário e a árvore de decisão para os dispositivos móveis quando estes são autenticados ou requisitam tais artefatos;

- sincronizar dados com o servidor inteligente: os servidores são sincronizados para atualizar questionário, árvore de decisão e dados de diagnósticos armazenados no banco de dados local.

\subsection{Servidor inteligente}

Este é um modulo centralizado que processa os dados fornecidos por cada unidade de saúde junto a uma base de dados já consolidada de determinado domínio da medicina. Esse processamento utiliza inteligência computacional para produzir a árvore de decisão utilizada pela aplicação móvel. O servidor inteligente também permite o armazenamento de scripts contendo árvores de decisão que não utilizem inteligência artificial. Suas principais funcionalidades são:

- autenticar servidor intermediário: o servidor recebe os dados de autenticação de cada servidor intermediário e retorna uma mensagem de validação. Dessa forma, aumenta-se a segurança ao permitir que apenas servidores autorizados acessem o servidor inteligente;

- gerenciar questionários de doenças: questionários de doenças são exibidos, modificados ou inseridos por profissionais de saúde;

- produzir árvore de decisão: a partir de uma base de dados relacionada a um questionário de doença, é feito o processamento no qual resulta em uma árvore de decisão que fundamenta o diagnóstico da doença;

- $\quad$ sincronizar dados com o servidor intermediário: os questionários e artefatos de IA são enviados para os servidores intermediários.

\section{Considerações finais}

Este trabalho apresentou a experiência de modelagem de sistema móvel de apoio ao diagnóstico médico utilizando equipamentos móveis. Este trabalho envolve diferentes equipes de diferentes áreas do conhecimento e este artigo buscou consolidar os conceitos e trazer resultados preliminares. A utilização de dispositivos móveis e inteligência computacional já é uma realidade em saúde, e sua aplicação neste trabalho reside na lacuna quanto ao seu uso em saúde básica como elemento de apoio à tomada de decisão baseada nas evidências coletadas, permitindo o uso do sistema por diferentes profissionais e procurando melhorar a qualidade do atendimento e da saúde da população. A visão de arquitetura e tecnologias com o apoio de profissionais de saúde constitui elemento importante para que o sistema atenda às partes interessadas e o recurso tecnológico seja realmente utilizado como serviço necessário, dentro dos padrões de projeto e informações reais de pacientes existentes.

Considerando a descentralização do serviço oferecido, o InteliMed é projetado para ser um sistema de suporte à decisão que permita o atendimento na casa do paciente, operando, assim, em ambiente remoto. É 
importante ressaltar que a conexão entre a aplicação móvel e o servidor intermediário é temporária e não é em tempo real, visto que há possíveis limitações de rede em áreas isoladas. Dessa forma, o uso como ferramenta de suporte à decisão é justificado pela carência de profissionais especializados, principalmente no interior dos estados.

Um aspecto importante na estratégia de desenvolvimento do InteliMed consiste na opção de focar, inicialmente, em uma doença e numa técnica de inteligência computacional específica que favoreça a construção da prova de conceito. A Asma foi definida pela sua relevância e alta incidência, o que possibilita a fácil aquisição de dados - característica relevante para utilização das técnicas de inteligência computacional -, bem como uma boa aplicabilidade da ferramenta pela alta demanda de diagnóstico desta doença. No entanto, a ideia é permitir o acoplamento de outras doenças, pelo perfil generalista da saúde básica. A definição da Árvore de Decisão como técnica a ser utilizada está fundamentada na sua simplicidade, o que amplia a viabilidade de aplicação prática da ferramenta e sua comprovada eficácia em projetos similares. Após a consolidação da ferramenta com o binômio Asma/Árvore de Decisão, serão definidas novas doenças e outras técnicas de inteligência computacional para ampliar o acervo do InteliMed.

\subsection{Estado atual do trabalho}

Algumas metas já foram alcançadas: foram estudados sistemas móveis já existentes relacionados à saúde; as tecnologias utilizadas em cada módulo foram selecionadas e diversos requisitos foram levantados e validados por profissionais que possuem contato com serviços de saúde comunitária.

Atualmente, a arquitetura da aplicação móvel está concluída enquanto a dos servidores está em desenvolvimento. Dessa forma, deu-se início à implementação do protótipo da aplicação móvel - o qual visa apoiar o diagnóstico de asma em crianças e adolescentes. Paralelamente, estão sendo definidas formas de representar e percorrer a árvore de decisão em dispositivos com baixo poder de processamento e pouca memória. Após finalizar a implementação, será possível obter resultados preliminares, assim como feedback dos usuários sobre o uso do sistema, possibilitando encontrar os pontos de melhoria da aplicação por meio de execução de experimentos e estudos de caso.

\subsection{Desafios encontrados}

O desenvolvimento para dispositivos móveis exige cuidados em diversos aspectos, tais como baixa capacidade de processamento e memória reduzida, assim como o meio de interação com o usuário e tamanho da tela são particulares de cada equipamento. Ainda, mostra-se um grande desafio representar e percorrer uma árvore de decisão - cujo tamanho é, a princípio, indefinido e variável entre as versões - em um dispositivo com baixo poder de processamento e memória reduzida.

Além dessas, outras dificuldades encontradas são: em relação ao desenvolvimento dos servidores, a pouca experiência, quando existente, da equipe de desenvolvimento em aplicações web torna-se um empecilho para o andamento do projeto. Também há questões relacionadas à segurança da informação, por exemplo, como transmitir os dados de forma íntegra e segura por meios inseguros, como a internet. Por fim, integrar equipes distribuídas fisicamente, visto que o projeto é formado por três equipes: uma responsável pelo módulo móvel; outra, pelo servidor inteligente e a última, pelos aspectos ligados à saúde.

\subsection{Trabalhos futuros}

Inicialmente, planeja-se desenvolver o InteliMed como prova de conceito para, em seguida, considerar os aspectos relacionados à usabilidade, segurança e padrões de informática em saúde. Dessa forma, é pretendido: (i) permitir um melhor uso de dispositivos móveis como ferramenta de apoio à decisão médica; (ii) treinar e educar profissionais de saúde para melhoria da qualidade assistencial; (iii) prover suporte a serviços de segunda opinião médica, integrando o sistema com hospitais e obtendo apoio de especialistas; (iv) ampliar o número de doenças suportada pela ferramenta e (v) ampliar a quantidade de técnicas de inteligência computacional utilizadas no suporte ao diagnóstico. 


\section{$7 \quad$ Agradecimentos}

Agradecemos à FINEP (Financiadora de Estudos e Projetos) e ao CNPq (Conselho Nacional de Desenvolvimento Científico e Tecnológico) pelo apoio financeiro recebido para execução deste projeto.

\section{Referências}

[1] GRASSO, M. Clinical Applications of Hand Held Computing. In: 17th IEEE Symposium on ComputerBased Medical Systems. Proceedings, 2004.

[2] SABBATINI, R. Telemedicina e Informatização em Saúde. In: Siqueira, E. (Org.). Technologies That Change Our Lives. São Paulo: Saraiva, 2007. Disponível em: $<$ http://www.sabbatini.com/renato/papers/InformatizacaoSaudeTelemedicina.pdf>.

[3] MINISTÉRIO DA SAÚDE, Departamento de Atenção Básica, Brasil. Disponível em: $<$ http://200.214.130.35/dab/>, 2010

[4] AMIT, G et al. Effects of computerized clinical decision support systems on practitioner performance and patient outcomes, a systematic review. Journal of the American Medical Association, 293(10), 2005, p. 1223 1238.

[5] $\mathrm{KOCH}, \mathrm{S}$. Home telehealth - Current state and future trends. International Journal of Health Informatics, v. 75 , Issue 8, 2006, p. 565-576.

[6] BAUMGART, D. Personal Digital Assistants in Health Care: experienced clinicians in the palm of your hand?. The Lancet, $\mathrm{n}^{\mathrm{o}}$ 366, 2006, p. 1210-22.

[7] CASTRO, L.S.S. et al. HandMed - Um Sistema Móvel Integrado para Captura Automática de Sintomas. In: CONGRESSO BRASILEIRO DE INFORMÁTICA NA SAÚDE, IX. Anais..., 2004.

[8] MORAES, D. A.; PISA, I. T.; LOPES, P. R. L. Protótipo para Coleta de Informações em Saúde Utilizando Dispositivos Móveis. In: CONGRESSO BRASILEIRO DE INFORMÁTICA NA SAÚDE, IX. Anais... 2004.

[9] MARTHA, A. et al. Clinic Web: PEP e interação com dispositivos móveis. In: CONGRESSO BRASILEIRO DE INFORMÁTICA NA SAÚDE, XI. Anais..., 2008.

[10] BREGA, J. et al. Levantamento Epidemiológico em Saúde Bucal Utilizando Ferramentas Móveis. In: CONGRESSO BRASILEIRO DE INFORMÁTICA NA SAÚDE, XI. Anais..., 2008.

[11] VIGOLO, M.; FADEL, F.; BASTOS, L. Coleta de Dados de Pacientes de Hanseníase via PDA. In: CONGRESSO BRASILEIRO DE INFORMÁTICA NA SAÚDE, XI. Anais..., 2008.

[12] CORREIA, R.; KON, F.; KON, R. Borboleta: A Mobile Telehealth System for Primary Homecare. In: 23rd ACM Symposium on Applied Computing. Proceedings, 2008.

[13] MEZAROBA, W.; MENEGON, M.; NICOLEIT, E. Registro Eletrônico de Paciente em uma UTI: Comunicação, Interação com Dispositivos Móveis e Previsão de Expansibilidade. In: CONGRESSO BRASILEIRO DE INFORMÁTICA NA SAÚDE, XI. Anais..., 2008.

[14] MACHADO, A.. et al. Utilização de Dispositivos Móveis, Web Services e Sotfware Livre no Monitoramento Remoto de Pacientes. In: CONGRESSO BRASILEIRO DE INFORMÁTICA NA SAÚDE, XI. Anais..., 2008

Revista Brasileira de Computação Aplicada (ISSN 2176-6649), Passo Fundo, v. 3, n. 1, p. 30-42, mar. 201141 
[15] LEE, V.; SCHNEIDER, H.; SCHELL, R.. Aplicações móveis: Arquitetura, projeto e desenvolvimento. São Paulo: Pearson Education do Brasil, 2005.

[16] SOCIEDADE BRASILEIRA DE PNEUMOLOGIA E TISIOLOGIA. IV Consenso Brasileiro no Manejo da Asma, 2006. Disponibilizado no Congresso Brasileiro de Pneumologia em Fortaleza, 2006.

[17] IV Diretrizes Brasileiras para o Manejo da Asma. J Bras Pneumol. 2006, 32(Supl 7):S 447-S 474.

[18] STERN T.; HUNT J.; NORTON H.J. Validating a Guidelines-Based Asthma Decision Support System: Step Two. Journal of Asthma, 46:933-935, 2009.

[19] CASTRO, L. K. K.; NETO, A. C.; FILHO, O. F. F. Prevalência de sintomas de asma, rinite e eczema atópico em escolares de 6 e 7 anos na cidade de Londrina (PR). J Bras Pneumol. 2010, 36(3):286-292.

[20] LEITE, M. et al. Avaliação do Questionário de Controle da Asma validado para uso no Brasil. J Bras Pneumol. 2008, 34(10):756-763.

[21] WANDALSEN, N. F. et al. Avaliação de critérios para o diagnóstico de asma através de um questionário epidemiológico. J Bras Pneumol. 2009, 35(3):199-205.

[22] WERK, L.N. et al. Beliefs about diagnosing asthma in young children. Pediatrics, v. 105, n. 3, March 2000.

[23] SOLÉ D. et al. International study of asthma and allergies in childhood (ISAAC) written questionnaire: validation of the asthma component among Brazilian children. J Invest Allergol Clin Immunol, 1998, 8:37682.

[24] GINA - Global Initiative for Asthma. Disponível em: <http://www.ginasthma.com/>, 2010.

[25] KEN, F. et al. A Tree-Based Decision Model to Support Prediction of the Severity of Asthma Exacerbations in Children. Journal of Medical Systems, v.34, p.551-562, 2010.

[26] OLIVEIRA, A.L.I. et al. A comparative Study of Machine Learning Techniques for Caries Prediction. 20th IEEE International Conference on Tools with Artificial Intelligence, 2008.

[27] Chae, Y. M. et al. Analysis of healthcare quality indicator using data mining and decision support system. Expert Systems with Applications, v. 24, p. 167-172, 2003.

[28] WITTEN, I. W; FRANK, E. Data Mining: Practical machine learning tools and techniques. 2nd. Edition, San Francisco: Morgan Kaufmann, 2005.

[29] GARCIA, S.C. O Uso de Árvore de Decisão na Descoberta de Conhecimento na Área da Saúde. 2003. Dissertação (Mestrado em Ciência da Computação) - Instituto de Informática, Universidade Federal do Rio Grande do Sul, Porto Alegre. 